\title{
Experiment on Flow Velocity Vector Measurement in EPs by Using 3D-PIV
}

\author{
Siyang Dai ${ }^{1,2}$, Yunxiao $\mathrm{Cao}^{2}$, Wenxiu Wang ${ }^{1}$ and Huanyu Zhao ${ }^{3,{ }^{*}}$ \\ ${ }^{1}$ School of Electrical Information, Dalian Jiaotong University, Dalian 116028, China \\ ${ }^{2}$ School of Electrical Engineering, Dalian University of Technology, Dalian 116024, China \\ ${ }^{3}$ Journal of Editorial Board, Dalian Minzu University, Dalian 116600, China \\ ${ }^{*}$ Corresponding author
}

\begin{abstract}
In the online-plate electrostatic precipitator(EPs), uses three-dimensional PIV technique and image processing technology to observe the fine particles of flow field under $-45 \mathrm{KV}$ pulse high pressure, and analyzes the motion state of the particles. Comparing with the traditional 2D-PIV technology, 3D-PIV technology can be more accurate and comprehensive to observe flow velocity vector of fine particles in electrode voltage. Avoiding inaccurate observation due to particles overlap. The experimental results show that the method can provide data support for more accurate description of particle motion behavior, which is great significance to improve the electrostatic precipitator.
\end{abstract}

Keywords-component; EPs; 3D-PIV; fine particles; flow velocity vector.

\section{INTRODUCTION}

The process of electrostatic precipitation(ESPs) is quite straightforward: applying high-voltage to make electrodes of different potential generate an electric field and create a ionized interelectrode gas in which suspended particles become charged and drift towards one electrode. Subsequently the suspended particles, dust will depose on the electrode plate. Now the EPs is an effective equipment of air pollution control in thermal power plant and other places because it could apply in the place with high temperature flue gas or large pressure, and it has less energy consumption, high efficiency of dust removal. But with the atmospheric control standards continue to improve, for example, "thermal power plant air pollutant discharge standards" (GB13223-2011) proposed air pollutant emissions below $30 \mathrm{mg} / \mathrm{m} 3$. The biggest problem with existing electrostatic precipitators is that the collection efficiency of fine particles (particle diameter $<10 \mu \mathrm{m}$ ) is small. The main reason is that when the high voltage is applied to the discharge electrode, the charged particles in the ionized gas flow to form the ion wind under the action of the external electric field, so that the dust produce complex turbulence effect, affecting the fine particles of the capture in EPs [1-2].

In recent years, domestic and foreign scholars have applied PIV technology to this field research. In 2001, first made use of the 2D-PIV technology to electrostatic precipitator, studying the device's internal flow field by Polish Academy of Sciences Mizeraczy [3]. In 2011, the French Poitiers University Noureddine Zouzou and other studies [2] have found that micro-particl-es formed three-dimensional motion state and turbulence effect in the ion wind.In 2015, Shen Xinjun et al[4] used 2D-PIV vis-ualization technique to studied the effect of ion wind on the movement of fine particles in the electrostatic precipitator and the trap of fine particles. In 2016, Cao Yunxiao et al. [5] proposed the use of image recognition to evaluate the fine particle capture efficiency.

\section{PIV TECHNOLOGY}

\section{A. 2D-PIV Technology}

Two-dimensional particle image velocity measurement technology (2D-PIV) can be traced back to the late 1970s, in recent decades has been continuously improved and development. The basic principle is to irradiate a plane of the flow field through the laser, and then add tracer particles in the flow field, the particle velocity represents the velocity of the fluid at the corresponding position.

It is structure and working principle of 2D-PIV (cf. Figure 1). A laser beam is used to illuminate a test plane in the flow field. The CCD camera synchronously records the position of the particles of secondary exposure adjacently. The velocity vector of the flow field, motion trajectories and particle density, distribution could obtained by the average displacement of each particle and the time interval of the exposure [4].

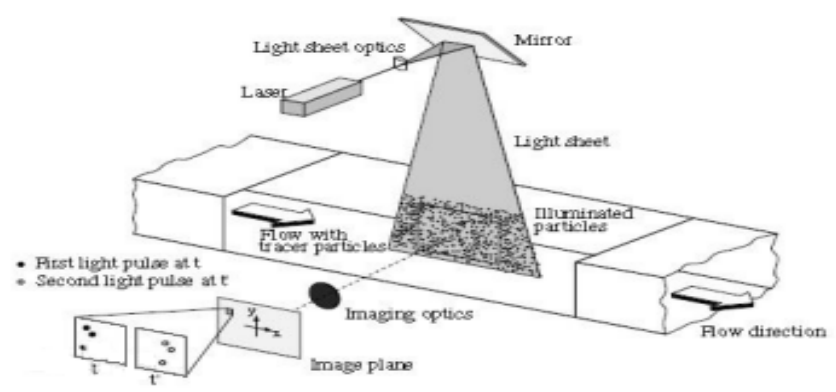

FIGURE I 2-D PIV TECHNOLOGY

\section{B. 3D-PIV Technology}

Fine particles researches of existing EPs are generally combined with 2D-PIV techniques and other techniques, but 2D-PIV only measures in-plane projections of threedimensional velocity vectors. there is an unavoidable error [6], 
such as particle overlap problem. This will not only increase the workload, and the measurement results and the actual results will produce some differences. The 3D-PIV system solves the shortcomings of 2D-PIV, which can reflect the actual state of the fine particles under the action of ion wind, and objectively restore the motion state of the flow field. The system uses two CCD cameras, the each camera's optical axis of system is perpendicular to the plane of observation window (see Figure 2).

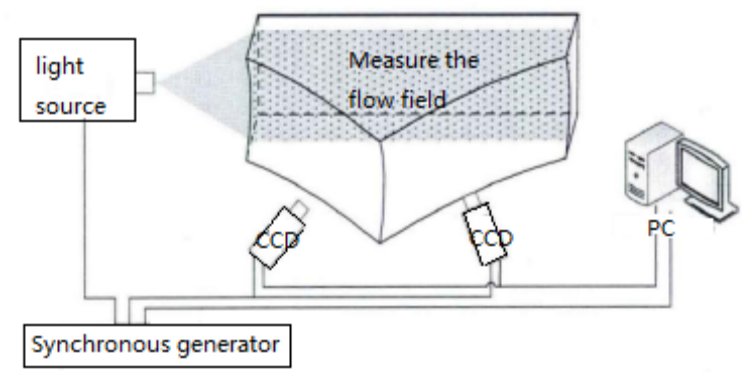

FIGURE II 3D-PIV TECHNOLOGY

The 3D-PIV system was built on the basis of the existing 2D-PIV system combined with image processing technology. The moving image of the fine particles in the electrostatic precipitator is obtained, and the motion state of the particles is objectively reconstructed by the image software, It is important to observe the dynamic information of fine particles, eliminate the interference of particles and the leakage of particles, calculate the efficiency of fine particle collection to improve the dust removal device.

\section{The Design of EXPerimental DeVice}

The device has been improved based on the laboratory existing device (see Figure 3), the device consists of two modules:Electrostatic precipitator module and 3D-PIV module. Electrostatic precipitator module includes electrostatic precipitator model, smoke cans, high voltage power supply, filter tube and fan and other components.

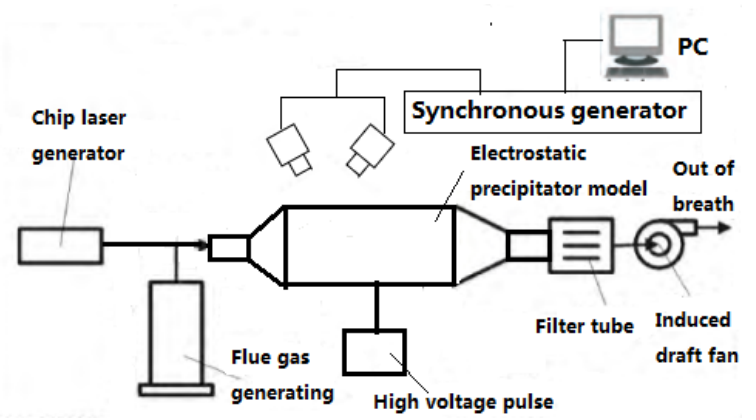

FIGURE III EXPERIMENTAL DEVICE

3D-PIV module includes synchronization controller, chip laser and two CCD cameras and other components. The thickness of the flake laser layer is $0.5 \mathrm{~mm}-1 \mathrm{~mm}$, which is flows from the inlet of the electrostatic precipitator with the direction of perpendicular to discharge electrode. The flue gas flows along the laser incident direction. The motion of fine particles can be clearly observed due to the diffuse of smoke particles. Camera selects SLR camera, the pixel is 8 million, shooting picture resolution for $3456 \times 2304$. The overlapping area of the two cameras is $70 \mathrm{~mm} \times 50 \mathrm{~mm}$. The camera is placed symmetrically at $90^{\circ}$, and the laser and two cameras are in the same plane to ensure good imaging results. For each camera, the The extension surface of shooting plane, imaging plane and lens plane need to intersect in a straight line to meet the Scheimpflug condition [7].

Generally the main body of the electrostatic precipitator is wired - plate, wire - cylinder and needle - plate and other structures, this paper selects the line-plate. Discharge electrode selects thorn line, the number of electrodes uses 7 (adjust the ran-ge of 1 to 7 ), thorn line and flue gas flow direction is perpendi-cular. Dust collection is made of white steel sheet 2, placed vertically in parallel. The main parameters of the electrostatic precipitator model are shown in Table 1.

TABLE I. MAIN PARAMETERS OF ESP MODEL

\begin{tabular}{|c|c|c|c|}
\hline \multirow{2}{*}{$\begin{array}{c}\text { Table } \\
\text { Head }\end{array}$} & \multicolumn{3}{|c|}{ Table Column Head } \\
\cline { 2 - 4 } & Parameter & Value & Unit \\
\hline \multirow{2}{*}{$\begin{array}{c}\text { Discharge } \\
\text { electrod }\end{array}$} & Round rod diameter & 4 & $\mathrm{~mm}$ \\
\cline { 2 - 4 } & Round length & 220 & $\mathrm{~mm}$ \\
\cline { 2 - 4 } & Thorn length & 8 & $\mathrm{~mm}$ \\
\hline \multirow{3}{*}{$\begin{array}{c}\text { Dust } \\
\text { collector }\end{array}$} & Length & 410 & $\mathrm{~mm}$ \\
\cline { 2 - 4 } & Wide & 200 & $\mathrm{~mm}$ \\
\cline { 2 - 4 } & 2 plates spacing & 180 & $\mathrm{~mm}$ \\
\hline \multirow{2}{*}{$\begin{array}{c}\text { Plexiclass } \\
\text { box }\end{array}$} & Plate thickness & 480 & $\mathrm{~mm}$ \\
\cline { 2 - 4 } & Length & 200 & $\mathrm{~mm}$ \\
\cline { 2 - 4 } & Wide & 300 & $\mathrm{~mm}$ \\
\hline
\end{tabular}

Tracer particles selected incense, the average diameter of less than $1 \mu \mathrm{m}$; high-voltage power supply $-45 \mathrm{kV}$ pulse high voltage, because compared with the DC voltage, pulse high voltage in the peak voltage $-45 \mathrm{kV}$ capture the highest efficiency, up to $91.23 \%$ [5]. Front pulse $<100$ ns, pulse width $<500$ ns.

Experiment first ignite the incense to produce flue gas, flue gas filled with smoke cans (about 120s), open the induced draft fan, control the fan to ensure that the movement of fine particles at $0.4 \mathrm{~m} / \mathrm{s}$. A burning incense burning time of about 4min, during this time to record the particles moving pictures.the pulse frequency is set to $100 \mathrm{~Hz}$ during the experiment.

\section{RESUlTS AND DisCUSSION}

Get the original image into imageJ software, set the display range, brightness, contrast and other parameters. Through the smoothing and other image preprocessing to do the preliminary processing work, through the differential algorithm to eliminate the impact of background noise, set the threshold to complete the binarization of the image processing, and then the mean filter and corrosion expansion, making the image can restore the actual image. The flow field velocity vector is shown in Fig. 4 , where the scale value indicates the velocity of the flow field in $\mathrm{m} / \mathrm{s}$. 

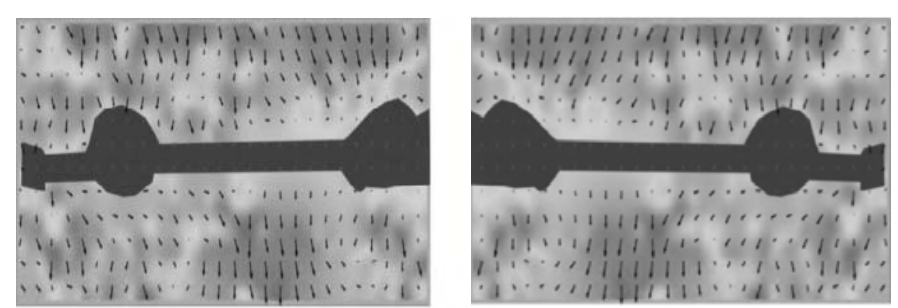

$\begin{array}{lllllllll}0.01 & 0.02 & 0.03 & 0.04 & 0.05 & 0.06 & 0.07 & 0.08 & 0.09\end{array}$

$\begin{array}{lllllllll}0.01 & 0.02 & 0.03 & 0.04 & 0.05 & 0.06 & 0.07 & 0.08 & 0.09\end{array}$

FIGURE IV FLOW VELOCITY VECTOR

\section{CONCLUSION}

In this paper, the 3D-PIV technique is used to collect the motion state of fine particles, and the problem of low efficiency of collecting fine particles is studied. Shoot image acquisition at $-45 \mathrm{KV}$ pulse high voltage. The dynamic sequence of the charged particles in the collected high voltage electric field is obtained by the cross correlation analysis. The results show that the 3D-PIV technique can describe the particle velocity, motion vector and law in a more direct and objective manner.

\section{ACKNOWLEDGMENT}

The authors would like to thank Dr. CaoYunxiao. of Dalian University of Technology for his part in the experiments and for the helpful discussions concerning the data treatment and analysis. Fund project:Dalian Minzu University Self - help Fund Project (DC2015020123).

\section{REFERENCES}

[1] Shen Xinjun, ZENG Yuxuan, Zheng Qinzhen, et al, "Measurements of flow field in wire-plate electrostatic precipitator during positive or negative corona discharge using PIV method," High Voltage Engineering. Hubei. China, vol. 40, pp. 2757-2763, September 2014.

[2] Noureddine Z, Dramane B, Moreau E, et al, "EHD flow and collection efficiency of a DBD ESP in wire-to-plane and plane-to-plane configurations”, IEEE Transactions on Industry Application, vol. 47, pp. 336-343, January 2011.

[3] Mizeraczyk J, Kocik M, Dekowski J, et al, "Measurements of the velocity field of the flue gas flow in an electrostatic precipitator model using PIV method,” Journal of Electrostatics. vol. 52, pp. 272-277, September 2001.

[4] Shen Xinjun.Study on Motion Laws and Removal Efficiencies of Fine Particles in Electrostatic Precipitator. China, Zhejiang University, 2015.

[5] Cao Yunxiao, Wang Zhiqiang, "Evaluation Method of Fine Particle Electrostatic Collection Efficiency Based on Image Identification”,High Voltage Engineering. Hubei. China, vol. 42, pp. 1455-1462, May 2016.

[6] Dannemann J, Pielhop K, “Cycle resolved multi-planar flow measurements in a four-valve combustion engine”, Experiments in Fluids. Vol. 50, pp. 961-976, April 2011.

[7] Piccinini R, Fernandes C, Andreotti M. Analysis of stereo scopic PIV measurements of in-cylinder flows using proper orthogonal decomposition, 13th Brazilian Congress of Thermal Sciences and Engineering, Uberlandia, MG, Brazil, pp. 1-10, 2010. 